\title{
The elliptical galaxy NGC 720: An unequal-mass galaxy merger remnant
}

\author{
S. B. Rembold ${ }^{1}$, M. G. Pastoriza ${ }^{1}$, and G. Bruzual ${ }^{2}$ \\ ${ }^{1}$ Instituto de Fisica, Universidade Federal do Rio Grande do Sul, Av. Bento Gonçalves 9500, Porto Alegre, Rio Grande do Sul, \\ Brazil \\ e-mail: rembold@if.ufrgs.br \\ 2 Centro de Investigaciones de Astronomía, AP 264, Mérida 5101-A, Venezuela
}

Received 1 December 2004 / Accepted 8 February 2005

\begin{abstract}
The stellar population of the central region of the galaxy NGC 720 has been investigated through longslit optical spectroscopy. The velocity dispersion and 13 Lick indices were obtained as a function of the radius along the semimajor axis of the galaxy. The $\mathrm{Mg}_{2}$ index presents a gradient of $\mathrm{dMg}_{2} / \mathrm{d} \log r=-0.079$ which behaves similarly to the Fe I lines, indicating no enhancement of $\mathrm{Mg}_{2}$ in relation to $\mathrm{Fe}$ I. The stellar population ages and metallicities were derived by a population synthesis method using available evolutive spectrophotometric models. The synthesis indicates a strong age gradient along the semimajor axis of NGC 720. In the central region a $13 \mathrm{Gyr}$ and solar metallicity stellar population dominates the flux at $5870 \AA$; the contribution of this component vanishes at a radius of $0.73 \mathrm{kpc}$, where the total flux is accounted for by a younger, $5 \mathrm{Gyr}$ and solar metallicity stellar population. For distances larger than $1 \mathrm{kpc}$ a $2.5 \mathrm{Gyr}$ component becomes very important. Moreover, NGC 720 is probably overabundant in $\mathrm{CN}$ in the center with respect to the solar abundance. The estimated total mass $\left(3.29 \times 10^{11} M_{\odot}\right)$ and the $\mathrm{Mg}_{2}$ gradient values suggest that this galaxy might have undergone a merger event. The correspondence between the $J$-band brightness profile decomposition and the result of the spectral synthesis shows that this galaxy is formed by an old (13-5 Gyr), bulge-like, small-scale and massive spheroid and a younger (5-2.5 Gyr), large-scale disk component. We discuss our results in comparison with available numerical simulations and propose that the merger event must have occurred about 4 Gyr ago.
\end{abstract}

Key words. galaxies: elliptical and lenticular, cD - galaxies: individual: NGC 720 - galaxies: kinematics and dynamics galaxies: stellar content

\section{Introduction}

The early-type isolated galaxy NGC 720 is a strong X-ray source (Fabbiano et al. 2002), and its X-ray emission distribution shows twisted isophotes. From the global X-ray emission, Buote et al. (2002) inferred that a flat, triaxial dark matter halo is present around this galaxy. However, the optical and infrared isophotes have a constant position angle (Jedrzejewski et al. 1987; Rembold et al. 2002). Also, numerous discrete, ultraluminous X-ray sources have been detected in this galaxy, a number comparable to those found in the Antennae and NGC 3256 merger systems (Jeltema et al. 2003). The spatial distribution of the discrete X-ray sources is in the form of shells, similar to those frequently observed in elliptical galaxies that are supposed to have undergone a recent merger with a disk or a dwarf galaxy (Barnes \& Hernquist 1992). On the other hand, shells and tidal features in the optical and infrared images of earlytype galaxies are more often found in isolated galaxies than in galaxies belonging to groups (Colbert et al. 2001). However, no optical shells have been detected in NGC 720. If we assume that this galaxy has suffered a merger in the past, according to Quinn (1984), after about $1.4 h_{70}^{-1}$ Gyr of the merger event, the shells should be detected no longer. The high $K$-band surface brightess fluctuation shown by this galaxy is another important constraint to its star formation history, once it is thought to be due to an intermediate-age population (Mei et al. 2001). This galaxy presents a luminosity profile in the infrared which cannot be fitted by a Sérsic law (Rembold et al. 2002). All these (sometimes contradictory) aspects of NGC 720 make it a real challenge to build up a single formation and evolution scenario for this object.

In this paper we investigate the spectral properties of the galaxy NGC 720 in order to constrain its stellar population properties and kinematics and clarify its formation and evolution. In Sect. 2 we present the observations and the data reduction; Sect. 3 describes the determination of the kinematical parameters from the spectra; in Sect. 4 we show the measurements of the spectral features; Sect. 5 presents the stellar population analysis. In Sect. 6 we discuss our results in view of 


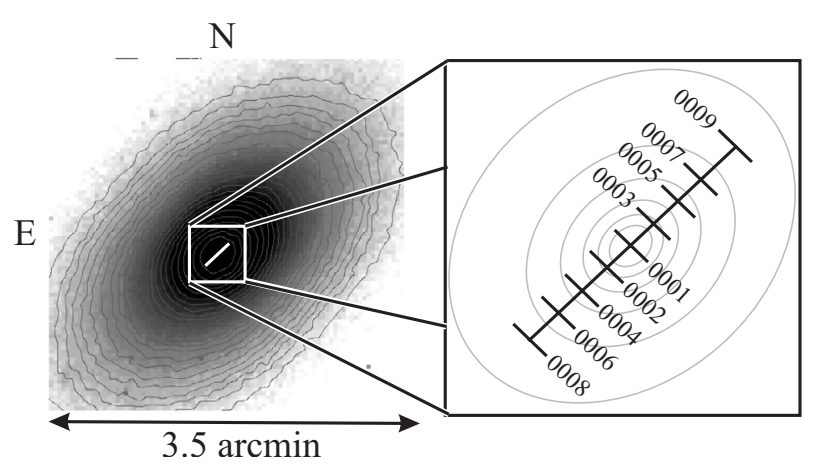

Fig. 1. Diagram showing the slit superimposed on a $J$-band image of NGC 720, and the position of each extraction.

a formation scenario for NGC 720. In Sect. 7 we present our conclusions.

\section{Observations and data reduction}

The observations were carried out at the ESO $1.52 \mathrm{~m}$ telescope in La Silla, Chile (September 2002). We used the Boller \& Chivens Spectrograph, configured to give spectra in the range 3500-7000 ^ with a spectral resolution of $2 \AA$ pix $^{-1}$ and spatial resolution of $0.86 \operatorname{arcsec~}_{\mathrm{pix}^{-1}}$. The longslit used was $0.5 \operatorname{arcsec}$ wide, and was oriented in the direction of the semimajor axis of the galaxy $\left(\mathrm{PA}=320^{\circ}\right.$; see Fig. 1$)$. The total integration time was $6000 \mathrm{~s}$, divided in 10 exposures.

The data reduction was made with the IRAF package and followed the typical reduction steps. The raw, two-dimensional spectra were flat-fielded (using exposures taken on twillight sky) and corrected for bad pixels, and 9 one-dimensional spectra were then extracted for each of the 10 two-dimensional frames, following the scheme shown in Table 1 . The 10 spectra corresponding to each spatial position were then mediancombined. The sky contribution was determined by extracting two spectra in the outermost regions of the two-dimensional frames ( 80 arcsec away from the center of the galaxy); these sky spectra were combined and subtracted from the other extractions (rescaled to each extraction width). A standard photometric star was also observed with the same setup, and a photometric calibration was obtained. Finally, a wavelength calibration was determined from observations of a He-Ne calibration lamp and applied to the spectra. We present in Fig. 2 the final spectra, corrected for radial heliocentric velocity $(z=$ 0.00582 ) and for Galactic extinction $A_{\mathrm{V}}=0.07 \mathrm{mag}$ (Schlegel et al. 1998).

\section{Kinematical data}

In order to determine the kinematical parameters from the spectra we have used an auto-correlation technique. An autocorrelation profile $F$ of a function $f$ is generated from

$F(z)=\int_{-\infty}^{\infty} f(\lambda) f(z-\lambda) \mathrm{d} \lambda$.

Hereafter, the variable $z$, corresponding to the "relative displacement" of the function $f$, will be referred to as a "channel".
Table 1. Extraction properties. ${ }^{\dagger}$ The negative sign represents an extraction SE of the center.

\begin{tabular}{lccc}
\hline \hline$r(\operatorname{arcsec})^{\dagger}$ & $r(\mathrm{kpc})$ & Width $(\operatorname{arcsec})$ & Width $(\mathrm{kpc})$ \\
\hline 0 & 0 & 2.46 & 0.36 \\
-2.46 & -0.36 & 2.46 & 0.36 \\
2.46 & 0.36 & 2.46 & 0.36 \\
-4.92 & -0.73 & 2.46 & 0.36 \\
4.92 & 0.73 & 2.46 & 0.36 \\
-7.38 & -1.09 & 2.46 & 0.36 \\
7.38 & 1.09 & 2.46 & 0.36 \\
-11.07 & -1.64 & 4.92 & 0.73 \\
11.07 & 1.64 & 4.92 & 0.73 \\
\hline
\end{tabular}

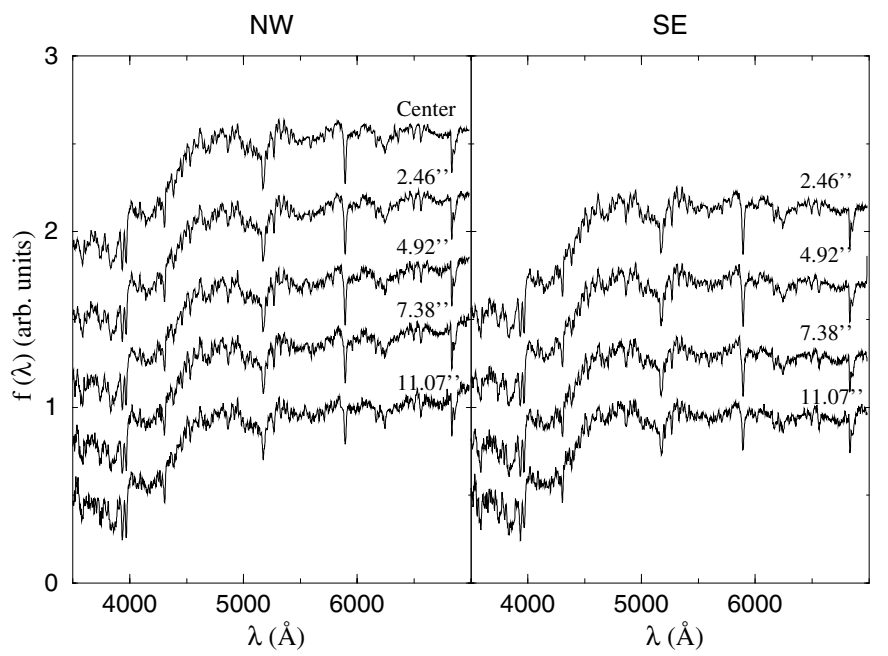

Fig. 2. Spectra of NGC 720 .

We expect that channels around zero correspond to high values of $F(z)$, and that $F(z)$ drops rapidly to some constant value for $|z| \gg 0$. It is convenient to renormalize the spectra in such a way that the auto-correlation profiles tend to zero for high channels; also, it is convenient to make the auto-correlation profile as sensitive as possible to the absorption lines and less sensitive to higher-scale patterns such as multiplets. To achieve that we have fitted 20th order polynomials to the continuum of the spectra, renormalized the spectra to this polynomial and subtracted a constant value (so that the average flux vanishes). We chose a 20th order polynomial because it is sensitive enough to describe the overall curvature of the spectra without changing the absorption lines. Since the broadening of a line is proportional to its wavelength, it is possible to associate a Gaussian convolution with a fixed dispersion when the spectra are transformed into a logarithmic wavelength scale. However, this process can result in loss of information. So, we have first resampled the spectra with cubic splines to a "resolution" of $0.35 \AA$ pix $^{-1}$, transformed the wavelength scale from $\lambda$ to $\ln (\lambda)$ and resampled again with a cubic spline, keeping the same resolution.

Next, we produced an auto-correlation profile for the galaxy spectra, and fitted the central correlation peak with a Gaussian profile. We also convolved the spectrum of a kinematic template G2IV star with Gaussians of different dispersions in steps of $5 \mathrm{~km} \mathrm{~s}^{-1}$, and produced an auto-correlation 
Table 2. Velocity dispersion data. The uncertainty in each individual determination is $5 \mathrm{~km} \mathrm{~s}^{-1}$.

\begin{tabular}{lc}
\hline \hline$r(\mathrm{kpc})$ & $\sigma\left(\mathrm{km} \mathrm{s}^{-1}\right)$ \\
\hline 0 & 250 \\
-0.36 & 235 \\
0.36 & 235 \\
-0.73 & 225 \\
0.73 & 225 \\
-1.09 & 215 \\
1.09 & 215 \\
-1.64 & 205 \\
1.64 & 205 \\
\hline
\end{tabular}

profile for each convolution, which was also fitted with a Gaussian profile. The convolved template spectrum whose auto-correlation profile better agrees with the galaxy autocorrelation profile gives the stellar velocity dispersion of the galaxy spectrum. The rotation velocity was derived by crosscorrelating the kinematic template spectrum and the galaxy spectrum: the channel corresponding to the peak of correlation gives the rotation velocity.

Table 2 shows the velocity dispersion data obtained. The stellar velocity dispersion presents a central peak of $250 \mathrm{~km} \mathrm{~s}^{-1}$ and decreases slowly to $\sigma=205 \mathrm{~km} \mathrm{~s}^{-1}$ at a radius of $3.29 \mathrm{kpc}$. The rotation velocity is very small (within the uncertainties); we can constrain its value to $v<50 \mathrm{~km} \mathrm{~s}^{-1}$. We estimate the $v / \sigma$ ratio to be 0.2 at $1.64 \mathrm{kpc}$ from the center, a value characteristic of dynamically-hot systems. Another determination of the $v / \sigma$ ratio is given by Binney et al. (1990), who found a value of 0.5 at $10 \mathrm{kpc}$ from the center, compatible with our value.

\section{Lick indices}

The wavelength range 3500-7000 ̊ contains many important spectral features. Several Fe I lines are present, as well as $\mathrm{Mg}_{2}$ and other molecular bands (CN, TiO II). The equivalent widths of the brightest absorption lines were measured following the definition of the Lick system (Faber et al. 1985), whose spectral windows are listed in Table 3. Prior to the measurements of equivalent widths and pseudocontinua, the spectra were normalized to the flux at $\lambda 5870 \AA$.

The spectral indices were corrected for line broadening using the calculated velocity dispersion for each extraction. We have measured the same spectral features in the kinematic template spectrum, convolved with Gaussians of different dispersions. The measured spectral feature $W_{\sigma}$ in the spectrum convolved with a dispersion $\sigma$, in comparison to the same feature measured in the unbroadened spectrum $W_{0}$, gives us a correction factor $C(\sigma)=W_{0} / W_{\sigma}$, which will be used to correct the corresponding feature measured in the galaxy spectrum.

Figure 3 and Table 4 show the corrected spectral indices, as well as the pseudocontinua fluxes. The uncertainties were derived through direct error propagation. The $\mathrm{Mg}_{2}, \mathrm{NaD}$ and TiO II lines present a strong gradient from the center to the outer regions as can be seen in Fig. 3. For $\mathrm{Mg}_{2}$ in particular we determine a gradient $\mathrm{d} \mathrm{Mg}_{2} / \mathrm{d} \log r=-0.079$; this gradient was determined by fitting a straight line to the $\mathrm{Mg}_{2}$ values as a function of $\log (r)$. The Fe I lines show a similar but less pronounced trend. It is interesting that the Fe I lines behave similarly to the $\mathrm{Mg}_{2}$ index, since many galaxies show an anticorrelation between these indices (Rickes et al. 2004; Carollo et al. 1993). The different slopes of the $\mathrm{Mg}_{2}$ gradient and that of the Fe I lines can be accounted for by an enhancement of $\mathrm{Mg}_{2}$ (Faber et al. 1992; Worthey et al. 1994). Therefore we conclude that NGC 720 has no relative enhancement of $\mathrm{Mg}_{2}$ to Fe I.

In order to describe the overall behaviour of the gradient of each spectral feature, we fit a modular function $y=a|x|+b$ to their distribution. This allows us to remove local variations in a particular extraction and to understand the main behavior of each spectral feature along the galaxy axis. These modular fits are superimposed in Fig. 3.

\section{Stellar population analysis}

The measured indices and pseudocontinua were used to detail the stellar population properties of NGC 720. In order to determine the metallicity and age of the stellar population in this galaxy we used the Bruzual \& Charlot (2003) evolutionary stellar population model. This model is based on a high-resolution library of observed stellar spectra which makes it possible to derive a detailed spectral evolution of single stellar populations (SSPs) at a resolution of $3 \AA$ between 3200 and $9500 \AA$, over a wide range of metallicities. There are many free parameters in these prescriptions, like the initial mass function (IMF), the attenuation by dust and the stellar evolution prescription. To limit the number of free variables in our analysis we fix the IMF to that of Salpeter (1955). Moreover, Bettoni et al. (2003) showed that the upper limit of projected dust concentration in NGC 720 is of only $4 M_{\odot} \mathrm{kpc}^{-2}$, so we do not expect a relevant attenuation by dust and fix $A_{\mathrm{V}}=0$. Also, we used the Padova 1994 tracks, as recommended by Bruzual \& Charlot (2003). We extracted from these models a set of 36 synthetic spectra covering a range of age and metallicity, whose properties are given in Table 5. Some template spectra, normalized to the flux at $5870 \AA$, are shown in Fig. 4.

\section{1. $\left[\mathrm{Mg}_{2} \mathrm{Fe}\right]-\mathrm{H} \beta$ diagnostic}

As a first step in our analysis we use the measured equivalent widths individually to constrain the metallicity and age of the stellar population of NGC 720. The success of this analysis will be maximized if we have two measured indices which are independently sensitive to metallicity and age of the stellar population. Based on the spectral evolution of SSPs of different metallicities, Bruzual \& Charlot (2003) defined an index which is strongly sensitive to the metallicity, poorly sensitive to age and very weakly dependent on $\alpha / \mathrm{Fe}$ :

$$
\left[\mathrm{Mg}_{2} \mathrm{Fe}\right]=0.6 \mathrm{Mg}_{2}+0.4 \log (\mathrm{Fe} 4531+\mathrm{Fe} 5015) \text {. }
$$

The index $\mathrm{H} \beta$, on the contrary, is much more sensitive to age than to metallicity. Thus, we can make a direct comparison between the observed $\left[\mathrm{Mg}_{2} \mathrm{Fe}\right]-\mathrm{H} \beta$ and the prediction of the models. 
Table 3. Definitions of the Lick system from Faber et al. (1985). Columns 3 and 4 give the pseudocontinua regions. * - Indices in magnitudes.

\begin{tabular}{lccc}
\hline \hline Index & Line/Band & $\Delta \lambda\left(\mathrm{PC}_{I}\right)$ & $\Delta \lambda\left(\mathrm{PC}_{S}\right)$ \\
\hline $\mathrm{Ca} \lambda 4227$ & $4223.500-4236.000$ & $4212.250-4221.000$ & $4242.250-4252.250$ \\
$\mathrm{G} \lambda 4300$ & $4282.625-4317.625$ & $4267.625-4283.875$ & $4320.125-4336.375$ \\
$\mathrm{Fe} \mathrm{I} \lambda 4383$ & $4370.375-4421.625$ & $4360.375-4371.625$ & $4444.125-4456.625$ \\
$\mathrm{Fe} \mathrm{I} \lambda 4531$ & $4515.500-4560.500$ & $4505.500-4515.500$ & $4561.750-4580.500$ \\
$\mathrm{H}_{\beta} \lambda 4861$ & $4848.875-4877.625$ & $4828.875-4848.875$ & $4877.625-4892.625$ \\
$\mathrm{Fe}$ I $\lambda 5015$ & $4979.000-5055.250$ & $4947.750-4979.000$ & $5055.250-5066.500$ \\
$\mathrm{Mg}_{2} *$ & $5155.375-5197.875$ & $4896.375-4958.875$ & $5302.375-5367.375$ \\
$\mathrm{Fe}$ I $\lambda 5270$ & $5247.375-5287.375$ & $5234.875-5249.875$ & $5287.375-5319.875$ \\
$\mathrm{Fe}$ I $\lambda 5406$ & $5390.250-5417.750$ & $5379.000-5390.250$ & $5417.750-5738.375$ \\
$\mathrm{Fe}$ I $\lambda 5709$ & $5698.375-5722.125$ & $5674.625-5698.375$ & $5724.625-5738.375$ \\
$\mathrm{Fe}$ I $\lambda 5782$ & $5778.625-5798.375$ & $5767.125-5777.125$ & $5799.625-5813.375$ \\
$\mathrm{NaD} \lambda 5895 *$ & $5878.625-5911.125$ & $5862.375-5877.375$ & $5923.875-5949.875$ \\
$\mathrm{TiO}^{*}$ & $6191.375-6273.875$ & $6068.375-6143.375$ & $6374.375-6416.875$ \\
\hline
\end{tabular}

Table 4. Measured equivalent line indices and continua (corrected for velocity dispersion effects); IN: equivalent widths (magnitudes for indices with *), UN: uncertainty of the index measurement, CO: continuum associated with the index.

\begin{tabular}{|c|c|c|c|c|c|c|c|c|c|c|c|c|c|c|}
\hline \multirow[t]{2}{*}{$\overline{r(\mathrm{kpc})}$} & & $\lambda 4227$ & $\lambda 4300$ & $\lambda 4383$ & $\lambda 4531$ & $\lambda 4861$ & $\lambda 5015$ & $\lambda 5177$ & $\lambda \overline{27270}$ & $\lambda 5406$ & $\lambda 5709$ & $\lambda 5782$ & 75895 & $\lambda 6237$ \\
\hline & & $\mathrm{CaI}$ & $\mathrm{CH}$ & $\mathrm{Fe} I$ & $\mathrm{Fe} I$ & $\mathrm{H} \beta$ & $\mathrm{Fe} I$ & $\operatorname{Mg}_{2}(*)$ & $\mathrm{Fe} I$ & $\mathrm{Fe} I$ & $\mathrm{Fe} I$ & $\mathrm{Fe} I$ & $\mathrm{NaD}(*)$ & TiO II $(*)$ \\
\hline \multirow[t]{3}{*}{0} & IN & 1.50 & 5.80 & 5.64 & 3.90 & 2.14 & 5.85 & 0.340 & 3.31 & 1.67 & 1.92 & 1.79 & 0.231 & 0.128 \\
\hline & UN & 0.21 & 0.35 & 0.44 & 0.32 & 0.21 & 0.45 & 0.007 & 0.25 & 0.22 & 0.18 & 0.16 & 0.009 & 0.005 \\
\hline & $\mathrm{CO}$ & 7.32 & 22.06 & 39.57 & 42.00 & 27.73 & 73.18 & 43.24 & 40.38 & 27.99 & 24.00 & 20.84 & 32.21 & 84.04 \\
\hline \multirow[t]{3}{*}{-0.36} & IN & 1.43 & 5.72 & 5.17 & 4.10 & 2.22 & 6.07 & 0.318 & 3.46 & 1.68 & 1.83 & 2.00 & 0.237 & 0.120 \\
\hline & UN & 0.25 & 0.42 & 0.54 & 0.39 & 0.25 & 0.55 & 0.007 & 0.31 & 0.27 & 0.22 & 0.20 & 0.011 & 0.006 \\
\hline & $\mathrm{CO}$ & 7.59 & 22.87 & 40.76 & 43.34 & 28.45 & 74.92 & 43.90 & 40.93 & 28.34 & 23.90 & 20.90 & 32.60 & 82.62 \\
\hline \multirow[t]{3}{*}{0.36} & IN & 1.55 & 5.64 & 5.61 & 3.85 & 2.13 & 5.87 & 0.339 & 3.22 & 1.70 & 1.83 & 1.86 & 0.240 & 0.120 \\
\hline & UN & 0.21 & 0.36 & 0.45 & 0.34 & 0.21 & 0.47 & 0.009 & 0.26 & 0.23 & 0.18 & 0.17 & 0.009 & 0.005 \\
\hline & $\mathrm{CO}$ & 7.18 & 21.35 & 38.71 & 40.47 & 26.87 & 70.62 & 41.87 & 38.85 & 27.22 & 23.46 & 20.57 & 32.42 & 83.85 \\
\hline \multirow[t]{3}{*}{-0.73} & IN & 1.50 & 6.02 & 4.64 & 4.17 & 2.58 & 5.80 & 0.312 & 3.10 & 1.54 & 1.84 & 1.97 & 0.220 & 0.117 \\
\hline & UN & 0.35 & 0.58 & 0.77 & 0.55 & 0.35 & 0.77 & 0.012 & 0.44 & 0.39 & 0.31 & 0.29 & 0.015 & 0.008 \\
\hline & $\mathrm{CO}$ & 7.62 & 22.89 & 40.20 & 43.40 & 28.50 & 74.58 & 43.48 & 40.29 & 27.91 & 23.46 & 20.53 & 32.24 & 80.83 \\
\hline \multirow[t]{3}{*}{0.73} & IN & 1.80 & 5.65 & 5.01 & 3.95 & 2.17 & 4.76 & 0.316 & 3.29 & 1.60 & 1.84 & 2.07 & 0.212 & 0.111 \\
\hline & UN & 0.37 & 0.63 & 0.83 & 0.59 & 0.38 & 0.85 & 0.012 & 0.47 & 0.40 & 0.32 & 0.29 & 0.015 & 0.008 \\
\hline & $\mathrm{CO}$ & 7.15 & 21.22 & 37.42 & 40.14 & 26.20 & 68.52 & 40.81 & 38.22 & 26.67 & 23.15 & 20.28 & 31.90 & 83.00 \\
\hline \multirow[t]{3}{*}{-1.09} & IN & 1.57 & 5.62 & 5.63 & 4.25 & 2.65 & 5.49 & 0.303 & 2.58 & 1.44 & 1.98 & 1.90 & 0.208 & 0.099 \\
\hline & UN & 0.40 & 0.68 & 0.87 & 0.64 & 0.41 & 0.90 & 0.014 & 0.52 & 0.45 & 0.36 & 0.34 & 0.017 & 0.010 \\
\hline & $\mathrm{CO}$ & 7.58 & 22.48 & 40.47 & 42.41 & 27.92 & 73.07 & 42.56 & 39.44 & 27.21 & 23.11 & 20.20 & 31.51 & 78.54 \\
\hline \multirow[t]{3}{*}{1.09} & IN & 1.04 & 5.18 & 4.42 & 4.73 & 2.25 & 4.60 & 0.300 & 3.16 & 1.47 & 1.79 & 1.86 & 0.174 & 0.116 \\
\hline & UN & 0.43 & 0.71 & 0.93 & 0.64 & 0.42 & 0.95 & 0.014 & 0.52 & 0.45 & 0.36 & 0.33 & 0.017 & 0.009 \\
\hline & $\mathrm{CO}$ & 7.28 & 21.87 & 38.48 & 41.60 & 26.95 & 70.29 & 41.70 & 38.89 & 27.16 & 23.48 & 20.56 & 32.24 & 84.16 \\
\hline \multirow[t]{3}{*}{-1.64} & IN & 1.22 & 4.89 & 4.68 & 3.84 & 2.58 & 4.57 & 0.273 & 2.59 & 1.60 & 1.86 & 1.65 & 0.173 & 0.088 \\
\hline & UN & 0.49 & 0.83 & 1.08 & 0.78 & 0.50 & 1.13 & 0.016 & 0.64 & 0.55 & 0.45 & 0.42 & 0.021 & 0.012 \\
\hline & $\mathrm{CO}$ & 7.96 & 23.34 & 41.00 & 43.69 & 28.65 & 73.85 & 43.12 & 39.86 & 27.97 & 23.48 & 20.44 & 32.42 & 80.59 \\
\hline \multirow[t]{3}{*}{1.64} & IN & 1.23 & 5.25 & 3.93 & 3.81 & 2.16 & 4.80 & 0.276 & 2.90 & 1.34 & 1.79 & 1.64 & 0.154 & 0.092 \\
\hline & UN & 0.51 & 0.86 & 1.14 & 0.81 & 0.53 & 1.17 & 0.017 & 0.66 & 0.57 & 0.45 & 0.42 & 0.021 & 0.012 \\
\hline & $\mathrm{CO}$ & 7.65 & 22.43 & 39.26 & 41.90 & 27.21 & 71.11 & 41.85 & 38.78 & 27.12 & 23.45 & 20.40 & 32.35 & 83.05 \\
\hline
\end{tabular}

Instead of making a direct comparison of the velocity dispersion corrected indices with the model predictions, we chose to "degrade" the resolution of the models by convolving the synthetic spectra with a Gaussian function corresponding to the central velocity dispersion of the galaxy. Figure 5 shows the $\left[\mathrm{Mg}_{2} \mathrm{Fe}\right]-\mathrm{H} \beta$ diagram for the observed spectra and for the degraded SSPs. Taking into account the error bars, our data are consistent with a stellar population with metallicity in the range $+0.0932<[\mathrm{Fe} / \mathrm{H}]<+0.5595$ and age in the range $2.5-13 \mathrm{Gyr}$. We can rule out a significant contribution of metal-poor or very young stellar populations.

We have tested the stability of this result with respect to the uncertainty of the measured velocity dispersion by convolving the synthetic spectra with Gaussians with slightly different 

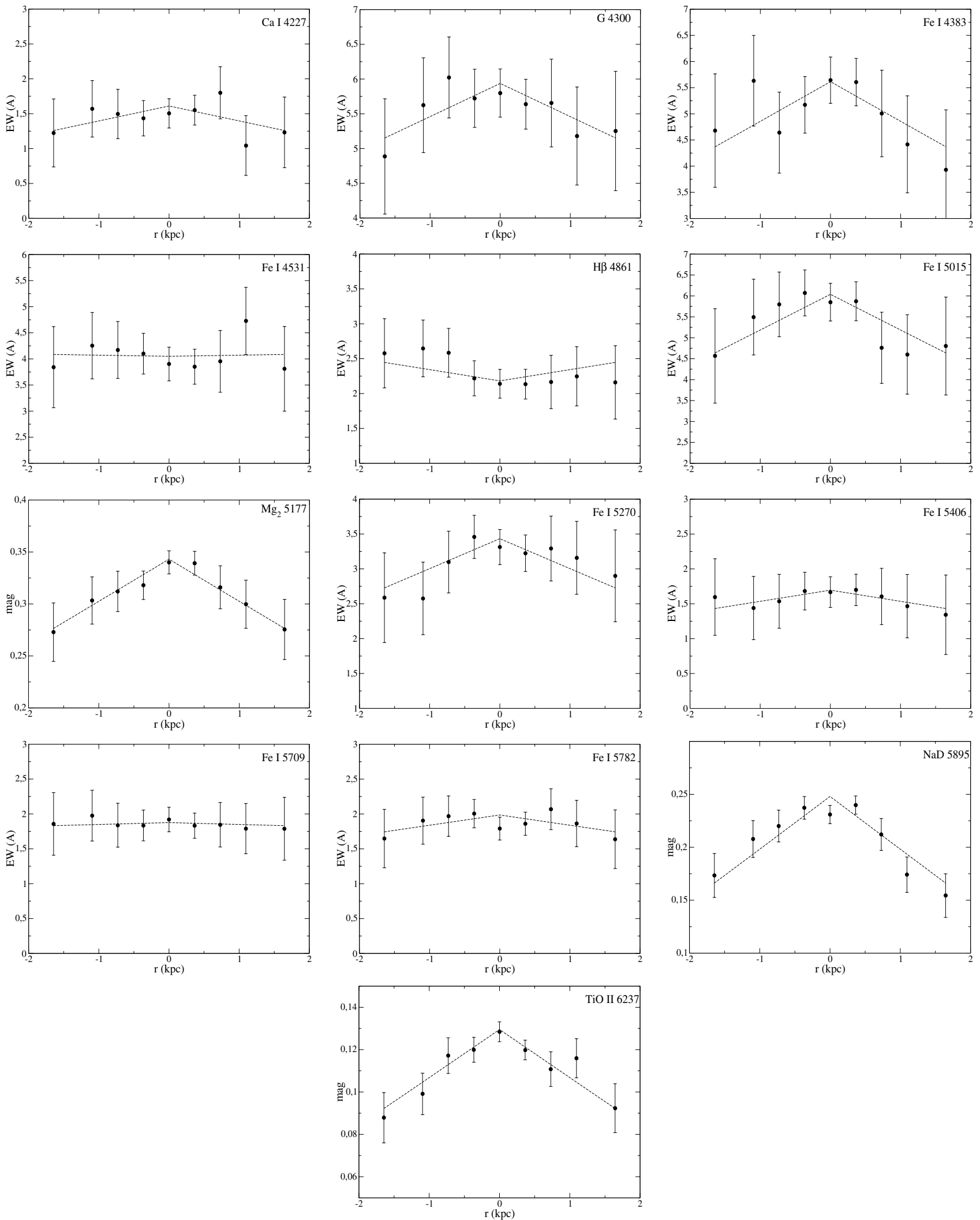

Fig. 3. Profiles of equivalent widths and magnitudes for absorption lines.

velocity dispersions $\left( \pm 5 \mathrm{~km} \mathrm{~s}^{-1}\right)$ and measuring the line indices. We found that the values vary less than $0.005 \AA$ in equivalent width for any index, much less than the uncertainty of the line indices measured on the galaxy spectrum. Therefore, the uncertainties in the velocity dispersion determination do not affect our results. 

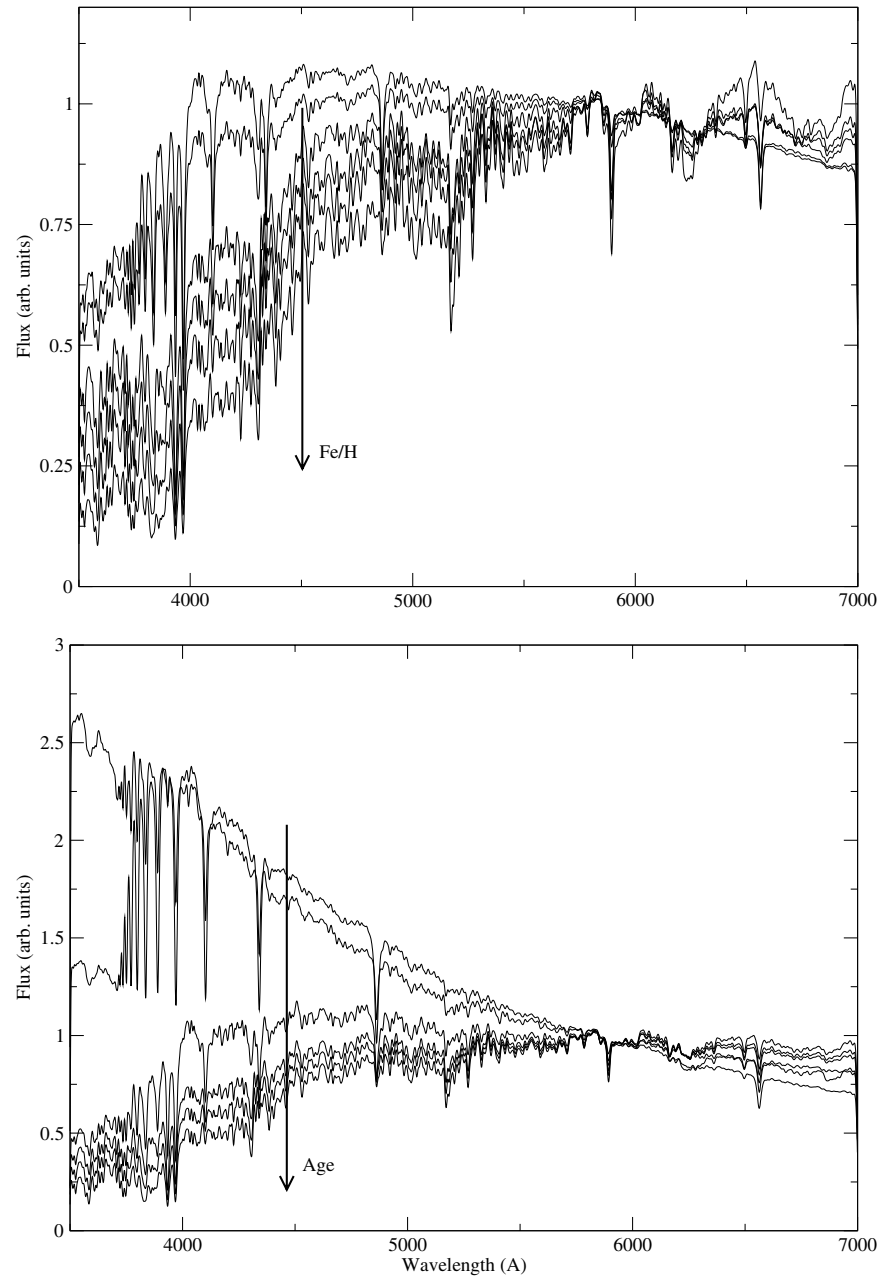

Fig. 4. Chosen template spectra with $13 \mathrm{Gyr}$ and metallicities from $\mathrm{m} 22$ to $\mathrm{m} 72$ (top diagram) and $\mathrm{m} 72$ metallicity from $13 \mathrm{Gyr}$ to $0.01 \mathrm{Gyr}$ (bottom diagram).

\subsection{Stellar population synthesis}

The next step in our analysis is to apply a stellar population synthesis method using as a basis the Bruzual \& Charlot (2003) synthetic spectra. The method consists of obtaining for each observed spectrum the linear combination that minimizes the following relation:

$\chi^{2}=\sum_{i=1}^{N}\left(\frac{I_{\mathrm{TOT}, i}-I_{\mathrm{MEA}_{i}}}{I_{\mathrm{TOT}, i}}\right)^{2}+\sum_{i=1}^{N}\left(\frac{P_{\mathrm{TOT}, i}-P_{\mathrm{MEA}_{i}}}{P_{\mathrm{MEA}, i}}\right)^{2}$

where $I_{i}$ are the spectral indices and $P_{i}$ the pseudocontinua. The total "synthetic" equivalent widths $I_{\mathrm{TOT}, i}$ are obtained from the equivalent widths of each template from

$I_{\mathrm{MEA}, i}=\frac{\sum_{j=1}^{36} w_{j} C_{j} I_{j}}{\sum_{j=1}^{36} w_{j} C_{j}}$,

where $C_{j}$ is the local pseudocontinuum of the line and $I_{j}$ is the equivalent width of the template $j$.

The computational algorithm varies the weights $w_{i}$ of each template from 0 to 1 in a given step and selects the set of weights that best reproduces the observed spectrum. Our previous analysis of the $\left[\mathrm{Mg}_{2} \mathrm{Fe}\right]-\mathrm{H} \beta$ diagram allows us to rule

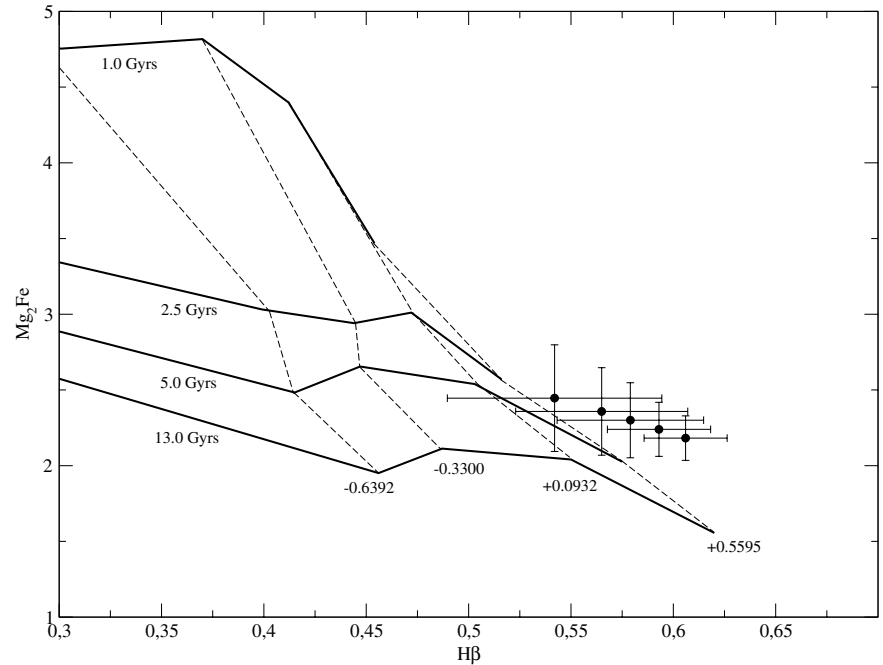

Fig. 5. $\left[\mathrm{Mg}_{2} \mathrm{Fe}\right]-\mathrm{H} \beta$ diagram showing the observed values superimposed on a set of models convolved on a Gaussian corresponding to the central velocity dispersion of NGC 720.

Table 5. Synthetic spectra from Bruzual \& Charlot (2003) models. Columns 2 and 3 give the metallicity and the age of each template.

\begin{tabular}{|c|c|c|}
\hline Synthetic spectrum code & {$[\mathrm{Fe} / \mathrm{H}]$} & Age (Gyr) \\
\hline m72_1300 & +0.5595 & 13.0 \\
\hline m72_0500 & +0.5595 & 5.0 \\
\hline m72_0250 & +0.5595 & 2.5 \\
\hline m72_0100 & +0.5595 & 1.0 \\
\hline m72_0010 & +0.5595 & 0.1 \\
\hline m72_0001 & +0.5595 & 0.01 \\
\hline m62_1300 & +0.0932 & 13.0 \\
\hline m62_0500 & +0.0932 & 5.0 \\
\hline m62_0250 & +0.0932 & 2.5 \\
\hline m62_0100 & +0.0932 & 1.0 \\
\hline m62_0010 & +0.0932 & 0.1 \\
\hline m62_0001 & +0.0932 & 0.01 \\
\hline m52_1300 & -0.3300 & 13.0 \\
\hline m52_0500 & -0.3300 & 5.0 \\
\hline m52_0250 & -0.3300 & 2.5 \\
\hline m52_0100 & -0.3300 & 1.0 \\
\hline m52_0010 & -0.3300 & 0.1 \\
\hline m42_0001 & -0.3300 & 0.01 \\
\hline m42_1300 & -0.6392 & 13.0 \\
\hline m42_0500 & -0.6392 & 5.0 \\
\hline m42_0250 & -0.6392 & 2.5 \\
\hline m42_0100 & -0.6392 & 1.0 \\
\hline m42_0010 & -0.6392 & 0.1 \\
\hline m42_0001 & -0.6392 & 0.01 \\
\hline m32_1300 & -1.6464 & 13.0 \\
\hline m32_0500 & -1.6464 & 5.0 \\
\hline m32_0250 & -1.6464 & 2.5 \\
\hline m32_0100 & -1.6464 & 1.0 \\
\hline m32_0010 & -1.6464 & 0.1 \\
\hline m32_0001 & -1.6464 & 0.01 \\
\hline m22_1300 & -2.2490 & 13.0 \\
\hline m22_0500 & -2.2490 & 5.0 \\
\hline m22_0250 & -2.2490 & 2.5 \\
\hline m22_0100 & -2.2490 & 1.0 \\
\hline m22_0010 & -2.2490 & 0.1 \\
\hline m22_0001 & -2.2490 & 0.01 \\
\hline
\end{tabular}




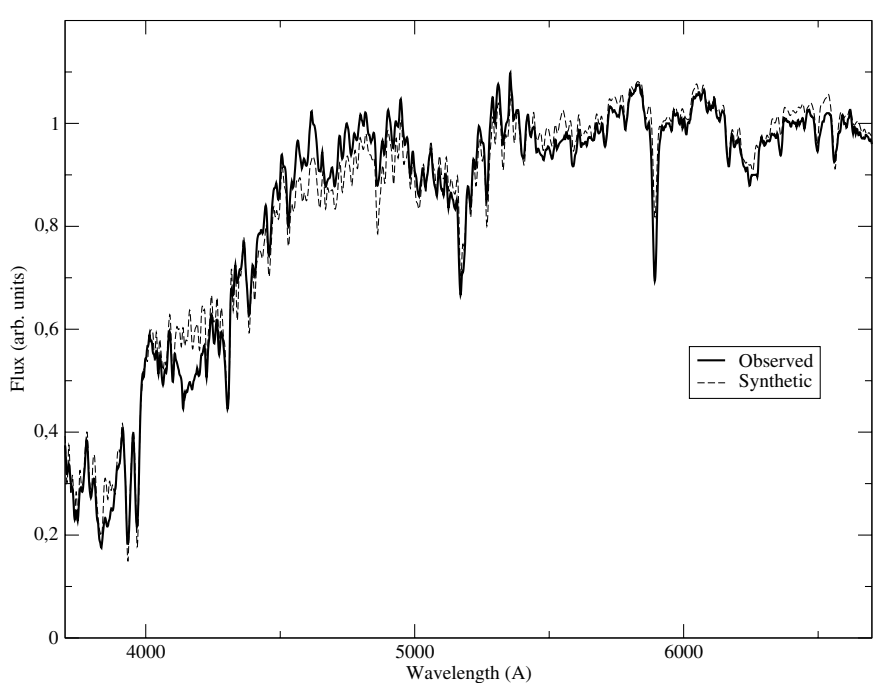

Fig. 6. Synthetic and observed central spectrum of NGC 720, normalized to the flux at $\lambda 5870 \AA$.

Table 6. Contribution of the templates to the flux at $5870 \AA$ A.

\begin{tabular}{lcccccc}
\hline \hline$r(\mathrm{kpc})$ & \multicolumn{3}{c}{$[\mathrm{Fe} / \mathrm{H}]=+0.5595$} & \multicolumn{3}{c}{$[\mathrm{Fe} / \mathrm{H}]=+0.0932$} \\
& $13 \mathrm{~Gy}$ & $5 \mathrm{~Gy}$ & $2.5 \mathrm{~Gy}$ & $13 \mathrm{~Gy}$ & $5 \mathrm{~Gy}$ & $2.5 \mathrm{~Gy}$ \\
\hline 0 & 0.000 & 0.000 & 0.000 & 0.707 & 0.293 & 0.000 \\
$+/-0.36$ & 0.214 & 0.000 & 0.000 & 0.202 & 0.584 & 0.000 \\
$+/-0.73$ & 0.000 & 0.000 & 0.000 & 0.000 & 1.000 & 0.000 \\
$+/-1.09$ & 0.000 & 0.000 & 0.000 & 0.000 & 0.875 & 0.125 \\
$+/-1.64$ & 0.000 & 0.000 & 0.000 & 0.000 & 0.419 & 0.581 \\
\hline
\end{tabular}

out templates with $[\mathrm{Fe} / \mathrm{H}] \leq-0.3300$ and also those that are younger than $1 \mathrm{Gyr}$; therefore a set of only 16 templates will be used a basis for the synthesis.

For each observed spectrum we run a first synthesis varying the $w_{i}$ s with a step of 0.05 . After this run, we eliminate the templates with zero contribution. A second run is performed with a step of 0.02 , and again the templates with zero contribution are discarded. The final results are reached in a third run with a step of 0.01. As input data for the synthesis we used the index values determined from the modular fits (Fig. 3) in order to follow the general behaviour of the indices. We have used all 13 spectral features and pseudocontinua to perform the synthesis as well as the $\left[\mathrm{Mg}_{2} \mathrm{Fe}\right]$ index. Therefore, there were 27 values to be fitted by the synthesis algorithm.

The synthesis result is listed in Table 6, where we present the percentage contribution of the templates to the total flux at $5870 \AA$. The synthetic spectrum of the central extraction and the observed central spectrum are plotted in Fig. 6 . The overall agreement is good, except in the $\mathrm{CN}$ band at $4200 \AA$. This departure, which is seen only in the innermost spectra, is probably caused by differences in the element abundance ratios between this galaxy and the Galactic stars used to build the Bruzual \& Charlot (2003) models. Enhanced abundances of $\mathrm{Mg}, \mathrm{C}$ and $\mathrm{N}$ have been invoked to account for the departures of models from observations of $\mathrm{CN}_{1}, \mathrm{CN}_{2}, \mathrm{C}_{2} 4668$ and $\mathrm{Mg}_{1}$. Consequently, this galaxy is probably overabundant in $\mathrm{C}$ and $\mathrm{N}$ in the center, with respect to the solar abundance. The synthesis result listed in Table 6 indicates a strong age gradient. In the central region a $13 \mathrm{Gyr}$ and solar metallicity stellar population dominates the flux at $5870 \AA$; the contribution of this component vanishes at a radius of $0.73 \mathrm{kpc}$, where the total flux is accounted for by a younger stellar population 5 Gyr old with solar metallicity. For distances larger than $1 \mathrm{kpc}$ a component of $2.5 \mathrm{Gyr}$ becomes very important.

Our synthesis results agree with the age gradient found by Trager et al. (2000). Concerning the metallicity, we do not observe the negative gradient found by Trager et al. (2000) and the supersolar abundance found by Terlevich et al. (2002). Since we have used 27 constraints in the synthesis, and the other authors have used less than 5 parameters, we consider our results more robust.

\section{Discussion}

The results of the stellar population synthesis together with the infrared photometric properties of NGC 720 can help us build a scenario of the structure and formation of this galaxy. The $J H K$ brightness profiles cannot be fitted by a single Sérsic law (Rembold et al. 2002). Using the $J$-band profile data from that work, we performed a new fit and found that a Sérsic+exponential law describes the profile well, as shown in Fig. 7. The structural parameters of the components are listed in Table 7. Up to 7 arcsec from the galaxy center, the bulge component dominates the infrared emission, while the disk component dominates the $J$ flux from 7 arcsecs on. Moreover, integrating both brightness laws up to $r \rightarrow \infty$ to get the total bulge $\left(J_{\mathrm{b}}=12.60 \mathrm{mag}\right)$ and disk $\left(J_{\mathrm{d}}=13.32 \mathrm{mag}\right)$ magnitudes separately and considering as an estimate that both subsystems have the same mass-to-light ratio, we find that the bulge is twice as massive as the disk.

On the other hand, Khosroshahi et al. (2000) found a strong correlation of the Sérsic exponent $n$ with $r_{\mathrm{e}}$ and $\mu_{\mathrm{e}}$ of the infrared brightness profiles of elliptical galaxies and bulges of spiral galaxies. This relation is given by

$\log n=0.172 \log r_{\mathrm{e}}-0.069 \mu_{\mathrm{e}}+1.18$.

Applying in this equation our $r_{\mathrm{e}}$ and $\mu_{\mathrm{e}}$ values derived from the $J$ band brightness profile, we find $n=0.84$, which agrees with the value obtained from our fit within the uncertainties. This agreement is a strong indication that our decomposition reflects a real detection of subsystems.

The histogram representing the percentage contribution to the flux at $5870 \AA$ of the $13 \mathrm{Gyr}, 5 \mathrm{Gyr}$ and $2.5 \mathrm{Gyr}$ templates is also shown in Fig. 7. The photometric profiles of the bulge and disk and the stellar population age gradient are linked to each other. The central bulge-like component is associated with the older (5-13 Gyr) stellar population, while the photometrically detected large-scale disk component has an age of 2.5-5 Gyr.

Carollo et al. (1993) have found a bimodal trend in the $\mathrm{Mg}_{2}$ gradient of early-type galaxies: for masses below $\approx 10^{11} M_{\odot}$, the $\mathrm{Mg}_{2}$ index gradient correlates with galaxy mass, while for higher masses this correlation disappears. This is interpreted as a result of the merging processes which form (at least part of) the higher mass systems and destroy the $\mathrm{Mg}_{2}$ gradients of the original galaxies. In order to compare our data with those of Carollo et al. (1993) we need to estimate the mass 


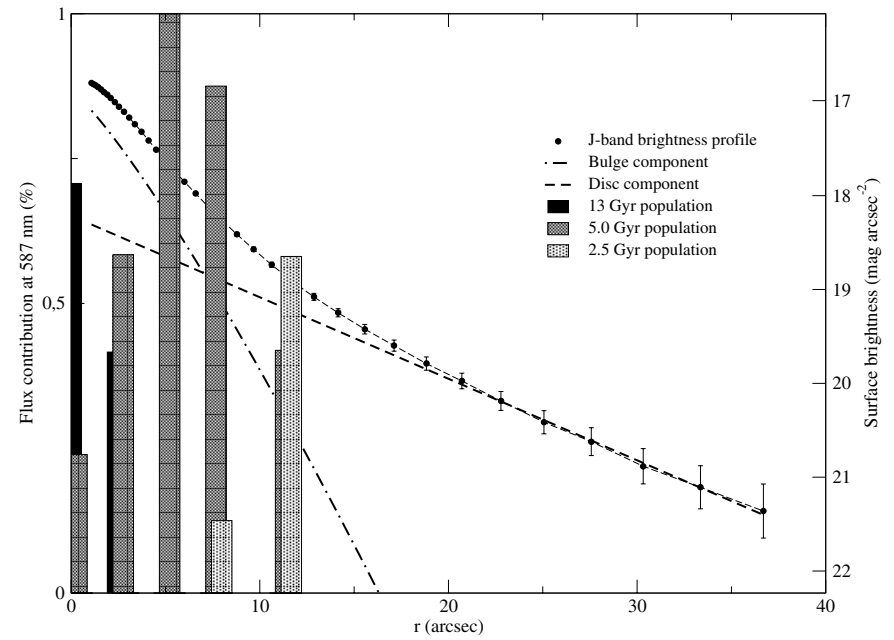

Fig. 7. Photometric $J$-band brightness profile of the galaxy NGC 720 and the percentage contribution of the templates to the total flux at $5870 \AA ̊$.

Table 7. Parameters of the best-fit brightess profile laws of the galaxy NGC 720. Column (1): effective radius of the Sérsic fit, in arcseconds. Column (2): effective surface brightness of the Sérsic fit, in mag $\operatorname{arcsec}^{-2}$. Column (3): exponent of the Sérsic profile (shape parameter). Column (4): effective magnitude of the disk (exponential) fit, in arcseconds. Column (5): effective surface brightness of the disk fit, in mag $\operatorname{arcsec}^{-2}$.

\begin{tabular}{ccccc}
\hline \hline$r_{\mathrm{e}}$ & $\mu_{\mathrm{e}}$ & $n$ & $r_{\mathrm{d}}$ & $\mu_{\mathrm{d}}$ \\
$(1)$ & $(2)$ & $(3)$ & $(4)$ & $(5)$ \\
\hline 2.819 & 17.43 & 0.83 & 12.86 & 18.09 \\
\hline
\end{tabular}

of NGC 720. The approximation used by Carollo et al. (1993), assuming a Jaffe law and an isothermal approximation for the density distribution cannot be used in this case because the mass distribution of NGC 720 does not follow this condition. van der Marel (1991) analysed in detail the velocity dispersion and radial velocity distributions of NGC 720 and estimated the mass-to-light ratio in the $B$ band as $\Upsilon_{B}=3.68 M_{\odot} / L_{\odot}$. Therefore we estimated the total mass of NGC 720 using the absolute $B$ magnitude $M_{B}=-21.9$ (van der Marel 1991) as $M_{\mathrm{TOT}}=3.29 \times 10^{11} M_{\odot}$. The mass and $\mathrm{Mg}_{2}$ values obtained for this galaxy do not follow the relation found by Carollo et al. (1993) (see their Fig. 11); on the contrary, they place NGC 720 in the region of systems which are supposed to have undergone a major merger event during their evolution.

In the dissipative collapse scenario, the disk component formed during the collapse process will absorb the angular momentum of the collapsing clouds. Therefore the kinematics of the disk will be dominated by the rotation velocity. Consequently, for this component the $v / \sigma$ ratio is expected to be larger than 1. In the case of NGC 720 the kinematics of the disc is dominated by the velocity dispersion, as we have shown in Sect. 3. Therefore, the kinematics of this galaxy does not agree with that expected for the dissipative collapse scenario.

Bournaud et al. (2004) have shown that disk galaxy mergers in the range of mass ratios 4:1-10:1 can result in the formation of a hybrid system with (a) a radial profile typical of spiral galaxies with a massive exponential disk and a central bulge; and (b) kinematical properties of elliptical galaxies with a velocity dispersion of the same order or larger than the rotation velocity. Both properties have been observed in NGC 720, supporting a scenario of unequal-mass merging for the formation of this galaxy. In particular, once the observed $v / \sigma$ ratio is below 1 at all radii, models with mass ratios around $4: 1$ seem to be more adequate. According to the merger simulations, strong asymmetries can still be seen in the merger remnant after $2 \mathrm{Gyr}$; the system will be fully relaxed only around $4 \mathrm{Gyr}$ after the beginning of the merger event. Since we do not observe asymmetries or tidal debris in NGC 720 we estimate a lower limit for the merger event of $4 \mathrm{Gyr}$. The main discrepancy between our observations and the models of Bournaud, Combes \& Jog (2004) concerns the spatial distribution of the young stellar population in the galaxy. These authors suggest that the star formation in the hybrid merger remnant may be strong in the central kpc but weak in the disk. On the contrary, we have detected a strong contribution of young stars $(2.5 \mathrm{Gyr}$ old) in the disk, and not in the nucleus. More detailed models concerning the ISM distribution may be needed in order to account for systems like NGC 720.

\section{Conclusions}

In this work the stellar population and kinematics of the galaxy NGC 720 have been investigated through optical longslit spectroscopy.

We have determined the velocity dispersion and rotation velocity of the galaxy. The velocity dispersion presents a central peak of $250 \mathrm{~km} \mathrm{~s}^{-1}$ and decreases slowly to $\sigma=205 \mathrm{~km} \mathrm{~s}^{-1}$ at a radius of $1.64 \mathrm{kpc}$. The rotation velocity is very low (within the uncertainties); we can constrain its value by $v<50 \mathrm{~km} \mathrm{~s}^{-1}$ up to $1.64 \mathrm{kpc}$.

We have measured 13 Lick indices. The $\mathrm{Mg}_{2}$ index presents a gradient of $\mathrm{d} \mathrm{Mg}_{2} / \mathrm{d} \log r=-0.079$ and the Fe I lines show a less steep gradient with a similar trend to $\mathrm{Mg}_{2}$. Therefore we conclude that NGC 720 does not have an enhancement of $\mathrm{Mg}_{2}$ with respect to $\mathrm{Fe}$.

A diagram $\left[\mathrm{Mg}_{2} \mathrm{Fe}\right]-\mathrm{H} \beta$ was used to compare our data with the models of Bruzual \& Charlot (2003). Taking into account the error bars, our data are consistent with a stellar population with metallicity in the range $+0.0932<[\mathrm{Fe} / \mathrm{H}]<+0.5595$ and age in the range of $2.5 \mathrm{Gyr}<$ age $<13 \mathrm{Gyr}$.

The stellar population synthesis indicates a strong age gradient along the semimajor axis of NGC 720. In the central region a stellar population of $13 \mathrm{Gyr}$ and solar metallicity dominates the flux at $5870 \AA$; the contribution of this component vanishes at a radius of $0.73 \mathrm{kpc}$, where the total flux is accounted for by a younger stellar population $5 \mathrm{Gyr}$ old with solar metallicity. For radii larger than $1 \mathrm{kpc}$, a component of $2.5 \mathrm{Gyr}$ becomes very important. In the central region the $\mathrm{CN}$ band shows a large departure from the models, while other metallic lines such as Fe I are well reproduced by solar metallicity models. This indicates that the center of NGC 720 is probably overabundant in $\mathrm{C}$ and $\mathrm{N}$ with respect to the solar abundance. The correspondence between the brightness profile decomposition and the result of the spectral synthesis of the galaxy NGC 720 
shows that there are two distinct population components forming this galaxy: an old (5-13 Gyr), bulge-like, small-scale and massive spheroid in the center, and a young (2.5-5 Gyr), largescale disk component around the bulge.

We have estimated the total mass of NGC 720 as $M_{\mathrm{TOT}}=$ $3.29 \times 10^{11} M_{\odot}$ from the work of van der Marel (1991). We find that the mass and the $\mathrm{Mg}_{2}$ gradient obtained for this galaxy do not follow the relation found by Carollo et al. (1993); on the contrary, these values place NGC 720 in the region of systems which are supposed to have undergone a major merger event. This scenario is also supported by the models of Bournaud et al. (2004), suggesting that this galaxy may be a hybrid remnant of an unequal-mass merger of mass-ratio around 4:1.

Acknowledgements. We are grateful to $\mathrm{Ch}$. Bonatto for his valuable help in the revision of this paper. We thank the support from the Brazilian institution CNPq(PRONEX 66.2088/1997-2).

\section{References}

Barnes, J. E., \& Hernquist, L. 1992, ARA\&A, 30, 705

Bettoni, D., Galletta, G., \& Garcia-Burillo, S. 2003, A\&A, 405, 5

Binney, J. J., Davies, R. L., \& Illingworth, G. D. 1990, ApJ, 361, 78

Bournaud, F., Combes, F., \& Jog, C. J. 2004, A\&A, 418, 27

Bruzual, G., \& Charlot, S. 2003, MNRAS, 344, 1000
Buote, D. A., Jeltema, T. E., Canizares, C. R., et al. 2002, ApJ, 577, 183

Carollo, C. M., Danziger, I. J., \& Buson, L. 1993, MNRAS, 265, 553

Colbert, J. W., Mulchaey, J. S., \& Zabludoff, A. I. 2001, AJ, 121, 808

Fabbiano, G., Kim, D.-W., \& Trinchieri, G. 1992, ApJS, 80, 531

Faber, S. M., Friel, E. D., Burstein, D., et al. 1985, ApJS, 57, 711

Faber, S. M., Worthey, G., \& Gonzáles, J. J. 1992, in IAU Symp. 149, ed. B. Barbuy, \& A. Renzini, 255

Jedrzejewski, R. I., Davies, R. L., \& Illingworth, G. D. 1987, AJ, 94, 1508

Jeltema, T. E., Canizares, C. R., Buote, D. A., et al. 2003, ApJ, 585, 756

Khosroshahi, H. G., Wadadekar, Y., Kembhavi, A., \& Mobasher, B. 2000, ApJ, 531, 103

van der Marel, R. P 1991, MNRAS, 253, 710

Mei, S., Kissler-Patig, M., Silva, D. R., et al. 2001, A\&A, 376, 793

Prugniel, Ph., \& Simien, F. 1997, A\&A, 321, 111

Quinn, P. J. 1984, ApJ, 279, 596

Rembold, S. B., Pastoriza, M. G., Ducati, J. R., et al. 2002, A\&A, 391, 531

Rickes, M. G., Pastoriza, M. G., \& Bonatto, Ch. 2004, A\&A, 419, 449 Salpeter, E. E. 1955, ApJ, 121, 161

Schlegel, D. J., Finkbeiner, D. P., \& Davis, M. 1998, ApJ, 500, 525

Trager, S. C., Faber, S. M., Worthey, G., et al. 2000, AAS, 119, 1645

Terlevich, A. I., \& Forbes, D. A. 2004, MNRAS, 330, 547

Worthey, G., Faber, S. M., Gonzáles, J. J., \& Burstein, D. 1994, ApJS, 94, 687 